\title{
UTILIZAÇÃO DA TÉCNICA POR COMPONENTES PRINCIPAIS (ACP) E FATOR DE ILUMINAÇÃO, NO MAPEAMENTO DA CULTURA DO CAFÉ EM RELEVO MONTANHOSO
}

\author{
RUBENS A. C. LAMPARELLI ${ }^{1}$, LUIZ NERY ${ }^{2}$, JANSLE V. ROCHA ${ }^{3}$
}

\begin{abstract}
RESUMO: O objetivo deste trabalho foi avaliar as informações obtidas das imagens do satélite Landsat/TM5, utilizando técnicas de Análise por Componentes Principais (ACP) e Fator de Iluminação oriundo de um Modelo de Elevação do Terreno, calculado a partir de imagens ASTER, no mapeamento de áreas de café em terreno montanhoso. As imagens utilizadas (três) foram corrigidas para o efeito da atmosfera e cobriram, temporalmente, o ciclo da cultura. Foram calculadas as componentes principais e escolhidas as duas primeiras, as quais possuíam 94\% das informações, para a definição das amostras. As amostras resultantes da ACP foram utilizadas na classificação supervisionada cujo resultado foi comparado com uma classificação convencional e uma classificação multitemporal convencional. A acurácia das classificações foi realizada por meio do cálculo da Exatidão Global e do Coeficiente Kappa, tendo como base uma máscara da área cafeeira da região. Os resultados mostraram que a técnica de ACP foi efetiva no estabelecimento de classes de iluminação, assim como na escolha das amostras, apesar de estas não terem representado a área efetivamente classificada. Em função disto, as classificações foram mais acuradas, principalmente aquela que considerou todos os pixels de cada imagem classificada individualmente pelo método da ACP, confirmando a importância do aspecto multitemporabilidade .
\end{abstract}

PALAVRAS-CHAVE: sensoriamento remoto, ASTER, correção atmosférica, normalização radiométrica, processamento digital de imagens.

\section{COFFEE CROP MAPPING USING PRINCIPAL COMPONENT ANALYSIS AND ILLUMINATION FACTOR FOR COMPLEX RELIEF}

\begin{abstract}
The main goal of this study was to evaluate the information produced from Landsat/TM5 images using Principal Component Analysis (PCA) and Illumination Factor built from Digital Elevation Model from ASTER images for coffee areas mapping in complex relief. Three Landsat images were used to monitor the crop cycle. The Principal Component Analysis was applied to the Landsat images and the two first components were chosen, responsible for $94 \%$ of the initial information, and used as a sample set for the supervised classification of those images. That classification was compared with a conventional supervised classification (sampled from Landsat reflectance images) and multitemporal conventional supervised classification (using the three images). The accuracies of the classifications were calculated by Kappa index of agreement and Global Accuracy, using a coffee mask as reference. The results have shown that PCA was very efficient in illumination class definition as well as in sample choice, despite the samples had not represented the area classified. Due to that, the accuracy has increased, specially the one considering all the pixels classified as coffee in each image using PCA samples, demonstrating the importance of the multitemporal aspect.
\end{abstract}

KEYWORDS: ASTER, atmospheric correction, radiometric normalization, digital image processing.

\footnotetext{
${ }^{1}$ Pesquisador, Doutor, Cepagri/Unicamp, Av. André Tosello, 209, Campinas - SP, rubens@cpa.unicamp.br.

${ }^{2}$ Eng $^{\mathrm{O}}$ Civil, M.Sc., Unicamp, Faculdade de Engenharia Agrícola, Av. Candido Rondon, 501, Campinas - SP, luisnery@uol.com.br.

${ }^{3}$ Eng ${ }^{\mathrm{o}}$ Agric., Prof. Dr., Unicamp, Faculdade de Engenharia Agrícola, Av. Candido Rondon, 501, Campinas - SP.

Recebido pelo Conselho Editorial em: 11-3-2010

Aprovado pelo Conselho Editorial em: 4-4-2011
} 


\section{INTRODUÇÃO}

As características topográficas de uma das principais regiões produtoras de café no sul de Minas Gerais, especificamente o município de Guaxupé, dita montanhosa, representam um forte obstáculo na elaboração de mapas que contenham a quantificação das áreas ocupadas por este tipo de cultura.

A utilização de modelos digitais de elevação como informação auxiliar na identificação da cultura de café, em região montanhosa (VIEIRA et al., 2004), tem-se tornado mais frequente em função do desenvolvimento de novos produtos orbitais aliados a produtos convencionais (TM/Landsat, SPOT, etc.) em estudos multitemporais. O trabalho de CORDERO-SANCHO \& SADER (2007) mostrou alto potencial de utilização do Sensoriamento Remoto quando aplicado em conjunto com dados de iluminação do terreno, na identificação de áreas de café na Costa Rica.

Uma característica importante a ser considerada em classificações digitais de imagens em áreas montanhosas é a iluminação em cada vertente. Apesar de ser utilizada em alguns casos, ela considera as variáveis do relevo decompostas em duas partes: declividade e aspecto. Por outro lado, há trabalhos que consideram estas variáveis, declividade e aspecto, como um fator somente, simplificando a análise. Neste contexto, baseado na técnica de processamento de imagens, há algoritmos que consideram o Modelo Digital de Elevação (MDE) acrescido das informações da geometria de visada de uma imagem orbital. O produto resultante desta combinação expressa as variações de iluminação ao longo do terreno e é chamado de Fator de Iluminação (FI). CANAVESI (2008) utilizou este conceito para identificar diferentes tipos de amostras de resposta espectral do eucalipto. A autora utilizou a técnica de Componentes Principais e Fator de Iluminação para classificar diferentes regiões, em áreas com plantio desta cultura, encontrando bons resultados ao correlacionar a resposta espectral com a produção de madeira. As Componentes Principais adquirem importância no âmbito do Sensoriamento Remoto porque possibilitam que informações redundantes, trazidas por diferentes bandas espectrais ocasionadas entre outras pelo sombreamento ou assinaturas espectrais semelhantes de alvos diferentes, sejam removidas através de uma transformação matemática (RICHARDS \& JIA, 2006). O princípio básico da transformação matemática resume-se no fato de que as componentes principais, através das matrizes de Covariância, procuram achar um conjunto de eixos que proporcionem menor variabilidade dentro das classes e alta variabilidade entre as classes (RICHARDS \& JIA, 2006).

Assim, o objetivo deste trabalho foi avaliar a efetividade da técnica de Componentes Principais em conjunto com o FI no mapeamento da cultura do café em área de relevo montanhoso.

\section{MATERIAL E MÉTODOS}

A área de estudo compreende o município de Guaxupé, estando localizado na região sul de Minas Gerais. O município apresenta como coordenadas geográficas o retângulo envolvente: $46^{\circ} 46^{\prime} 6,2^{\prime \prime} \mathrm{O} / 21^{\circ} 15^{\prime} 0,0^{\prime \prime} \mathrm{S}$ e $46^{\circ} 37^{\prime} 23,56^{\prime \prime} \mathrm{O} / 21^{\circ} 4^{\prime} 40,82^{\prime \prime} \mathrm{S}$.

A título de referência, foi utilizada, neste trabalho, uma máscara de áreas com café, na região estudada. A máscara de café é composta por polígonos mostrando a distribuição espacial das áreas ocupadas com a cultura. As áreas foram espacializadas por meio da interpretação visual de uma imagem do satélite Ikonos-II obtida em fevereiro de 2002 e resolução espacial de 1 metro. Os pontos de dúvidas foram checados em levantamentos de campo. Esta máscara, que é um mapa temático binário, onde 1 são áreas de café, e zero, outros usos, foi utilizada como se fosse a referência de campo, dada sua confiabilidade, na etapa de checagem da qualidade do mapeamento das áreas de café obtidas pela metodologia proposta neste trabalho, que será descrita a seguir.

Dentre os 4.047 talhões contidos na máscara de café, cedido pela COOXUPÉ (Cooperativa Regional de Cafeicultores em Guaxupé Ltda.), 97 talhões são de lavouras de café com plantio adensado, perfazendo 127 hectares e 1.291 talhões distribuídos em 1.445 hectares de café adulto, 
objeto de levantamento topográfico cadastral realizado por meio de rastreamento com GPS diferencial. Estes talhões foram utilizados como referência terrestre.

O monitoramento da cultura por sensoriamento remoto foi realizado através de imagens Landsat 5. As imagens foram escolhidas para coincidir com algumas fases importantes do ciclo vegetativo, mais especificamente na segunda fase (indução, crescimento e dormência das gemas florais), na terceira fase (florada, chumbinho e expansão do fruto) e na quinta fase (maturação dos frutos), baseado nas fases fenológicas do cafeeiro, segundo CAMARGO \& CAMARGO (2001). As imagens utilizadas foram do sensor TM do satélite Landsat 5 na órbita/ponto 219/75 e uma imagem do sensor ETM+(Enhanced Thematic Mapper +)/Landsat 7 (data 7-6-2002) em todas as bandas do visível, infravermelho próximo e médio (Tabela 1).

TABELA 1. Descrição das imagens e algumas informações técnicas. Images characteristics and some technical information.

\begin{tabular}{cccc}
\hline Órbita Ponto & Satélite/Sensor & Data & Elevação/Azimute Solar \\
\hline $219 / 75$ & Landsat/TM & $15-8-2001$ & $40,1 / 47,2$ \\
$219 / 75$ & Landsat/TM & $5-12-2001$ & $60,4 / 97,5$ \\
$219 / 75$ & Landsat/TM & $12-4-2002$ & $44,4 / 52,8$ \\
$219 / 75^{*}$ & Landsat/ETM+ & $7-6-2002$ & ------------ \\
\hline
\end{tabular}

* Utilizada no processo de Normalização.

Foi também utilizada imagem do sensor ASTER instalado na plataforma Terra, adquirida nas bandas 3B e 3N, no intervalo de comprimentos de ondas de 0,78 a $0,86 \mu \mathrm{m}$. A imagem foi processada utilizando-se do software AsterDTM 2.2, um plug-in do software ENVI. O produto ASTER resultante permitiu a geração de um Modelo Digital de Elevação (MDE). Este produto foi utilizado em conjunto com as informações de posição do imageador em relação ao Sol para dar origem ao produto denominado Fator de Iluminação (CANAVESI, 2008). A imagem contendo a distribuição da iluminação refletida foi convertida para um intervalo variando de 0 (zero) a 1 (um), em que o primeiro valor corresponde ao pixel de menor brilho, e o segundo, o de maior brilho. LANG \& WELCH (1999), analisando os parâmetros estabelecidos para o valor cartográfico do modelo gerado pelas imagens ASTER, concluíram que são de suficiente qualidade os mapas na escala 1:50.000, que é a escala de referência dos mapas desta pesquisa.

Os trabalhos de campo foram realizados com o objetivo de coleta de pontos de controle para a verificação do posicionamento geográfico da máscara de café fornecida pela COOXUPÉ, e coleta de pontos para o registro das imagens TM/Landsat 5 e ETM+/Landsat 7.

A metodologia foi focada na análise multitemporal de imagens que cobrissem o ciclo vegetativo do café. O trabalho posterior à aquisição das imagens consistiu na transformação dos valores de energia radiante referente a cada pixel, codificadas em níveis de cinza, e proporcionais à radiância do mesmo pixel, em valores de reflectância que, segundo PONZONI \& SHIMABUKURO (2007), são valores mais adequados para a caracterização espectral dos alvos, utilizando diferentes bandas ou diferentes sensores, quando comparado ao valor digital.

Para ser possível o monitoramento temporal da cultura por sensoriamento remoto, este trabalho realizou três etapas fundamentais: definição da imagem de referência (ETM+) para ser utilizada na técnica de normalização MAD (Multivariate Alteration Detection); correção geométrica das imagens TM e ETM+, e aplicação da técnica de normalização nas imagens TM.

O aplicativo denominado SCORADIS - Sistema Computacional de Correção Radiométrica, desenvolvido por ZULLO JR. (1994), foi utilizado para a realização da transformação de níveis de cinza para valores de energia como passagem para a obtenção dos valores de quantidade de radiação refletida pelo alvo considerado por PONZONI \& SHIMABUKURO (2007), como o Fator Reflectância Bidirecional (FRB) para a imagem do ETM+/Landsat 7. Desta maneira, foram inseridas as informações de valores de espessura óptica de aerossóis, de coluna total de vapor 
d'água e de carga total de ozônio, obtidos dos produtos MOD 04, MOD 05 e MOD 07, derivados dos dados obtidos pelo sensor MODIS/ Plataforma Terra, referentes à mesma data de aquisição da imagem do ETM+/Landsat 7, conforme NASCIMENTO \& ZULLO JR. (2005).

Posteriormente, foi realizada a correção geométrica das imagens ETM+e TM, e, para isto, utilizou-se de coordenadas obtidas por GPS (Global Positioning System) para referenciar os pontos identificáveis na imagem e denominados pontos de controle, utilizando-se de equações polinomiais de primeiro grau, para evitar ao máximo a perda de informações espectrais. Também foi realizada uma inspeção da compatibilidade geométrica entre o mapa de polígonos (máscara) de café com as imagens ETM+ e TM do Landsat. A verificação consistiu na observação da existência de variações quanto ao registro da imagem Ikonos, o que envolveu a utilização dos mesmos pontos de controle rastreados com GPS e utilizados para o registro das imagens Landsat, resultando em distorções não significativas devido à diferença de resolução espacial entre as cenas Ikonos e as imagens do TM/Landsat 5 ( 1 e $30 \mathrm{~m}$, respectivamente).

Para que as imagens tivessem padrões espectrais comparáveis, foi aplicada a técnica MAD (Multivariate Alteration Detection) proposta por CANTY et al.(2004) como passo seguinte aos processos de correção atmosférica que resultou em cenas com informações de FRB de superfície. Neste processo, a imagem ETM+ foi utilizada como imagem de referência, e as imagens geradas pelo sensor TM foram utilizadas como alvo.

Para avaliar o processo de normalização, os valores da média e da variância das bandas da imagem que foi normalizada, foram comparados com os valores da média e da variância da imagem de referência, conforme metodologia adotada por MERCANTE (2008).

\section{Fator de iluminação}

O Fator de Iluminação foi obtido a partir de imagens ASTER com valores de pixel variando de zero (0) para locais sem iluminação, até um (1) para locais com máxima iluminação, denominada de Fator de Iluminação (FI). No cálculo da imagem Fator de Iluminação (FI), foi utilizada a Equação 1:

$$
\mathrm{FI}=\left(\left(\left(\cos \left(\varphi_{\mathrm{x}, \mathrm{y}}-\varphi_{\mathrm{s}}\right)\right)^{2}+\left(\cos \left(\theta_{\mathrm{x}, \mathrm{y}}-\theta_{\mathrm{s}}\right)\right)^{2}\right)^{1 / 2}\right.
$$

em que,

$$
\begin{aligned}
& \varphi_{\mathrm{x}, \mathrm{y}}-\text { orientação das vertentes, imagem azimutal; } \\
& \varphi_{\mathrm{s}} \text { - azimute solar; } \\
& \theta_{\mathrm{x}, \mathrm{y}}-\text { declividade, imagem zenital, e } \\
& \theta_{\mathrm{s}}-\text { zênite solar no momento da aquisição da imagem. }
\end{aligned}
$$

Por meio desta técnica, implementada no software Idrisi, construiu-se três (3) imagens FI referentes às datas de aquisição do TM/Landsat 5. Sobre essas imagens, contendo o intervalo máximo de valores de FI de 0 até 1 , foi aplicado o processo de fatiamento (slice) com o objetivo de

\begin{tabular}{|c|c|}
\hline \multicolumn{2}{|c|}{ Distribuição das Classes de Iluminação } \\
\hline Classes & Intervalo de Iluminação \\
\hline$\overline{\text { Escuro (sombreado) }}$ & 0,000 até 0,4453 \\
\hline Pouco iluminado & 0,4454 até 0,6023 \\
\hline Iluminado & 0,6024 até 0,7785 \\
\hline Muito iluminado & 0,7786 até 1,0000 \\
\hline
\end{tabular}
separar, em intervalos, cada uma das quatro classes (Tabela 2) de iluminação adotadas: Escuro (sombreado), Pouco Iluminado, Iluminado e Muito Iluminado.

TABELA 2. Relação entre as classes e intervalos de iluminação. Relationship between Class and illumination ranges. 
O número de classes de iluminação adotado foi definido após a realização de testes variando o número de intervalos que possibilitassem, em cada classe, identificar a maior quantidade possível de pixels, localizando-se na área de lavouras de café, tendo como base as áreas de café cadastradas (máscara).

Com o objetivo de utilizar ao máximo as informações disponíveis por meio da aplicação da técnica de ACP, após a preparação das imagens de Fator de Iluminação nos intervalos selecionados e com a sobreposição dos polígonos de café cadastrados, iniciou-se o processo de seleção de amostras de café adulto e café adensado nas condições de iluminação propostas. Para isso, foram considerados plantios adensados aqueles com espaçamentos de rua variando entre $1,5 \mathrm{~m}$ e $2,5 \mathrm{~m}$ e entre linhas $0,5 \mathrm{~m}$ a 1,0 $\mathrm{m}$. Os espaçamentos superiores aos adotados para plantio adensado foram definidos como espaçamentos para a classe de café adulto.

Em cada imagem de FI, foram selecionadas 212 amostras possíveis de identificação, sendo que, para as imagens TM/Landsat 5, de 15-8-2001 e 12-4-2002, os conjuntos de amostras referentes aos tipos de plantio de café ajustaram-se ao número de classes de iluminação. Para a imagem de 5-12-2001, as classes obtidas foram somente as do tipo Iluminado e Muito Iluminado, devido ao ângulo de elevação solar muito elevado na data de aquisição dessa imagem.

As amostras coletadas em cada imagem FI, para os tipos de plantio adensado e adulto, em cada classe de iluminação, foram organizadas de forma a conter as informações necessárias, a partir dos valores de FRB de superfície das amostras, para o processamento. No processamento, foi utilizado o software SPSS (Statistical Package for the Social Sciences) versão 16.0.2, na geração dos valores referentes aos autovetores, autovalores e os fatores $f 1$ e $f 2$, que nada mais são do que os novos eixos PC1 e PC2, das Componentes Principais.

Foram então criados gráficos para melhor visualização da distribuição dos valores no novo espaço de atributos em que as informações não são correlacionadas. E desta formação, foram escolhidas as amostras perfazendo 65 de cada tipo de plantio de café coletadas em cada classe de iluminação, formando assim as áreas de treinamento.

Para a etapa posterior à classificação, foi adotada a Distância de Mahalanobis, que possui como vantagens, sobre os classificadores como paralelepípedo e máxima verossimilhança, o fato de ser mais rápido, levar em conta a matriz de covariância e a sensibilidade do grau de direção ao qual as amostras se distribuem no espaço espectral.,sendo preservadas (RICHARDS \& JIA, 2006 ).

Para medir a efetividade da aplicação da técnica do Fator de Iluminação como estratégia para melhorar o mapeamento do café, foi estabelecido que três tipos de processamento seriam necessários para análise dos dados:

Técnica 1 - Utilização de classificação supervisionada com o algoritmo Distância de Mahalanobis a ser aplicada a cada uma das três imagens TM escolhidas. As amostras foram selecionadas (grupos de 65 amostras) das Componentes Principais. Esta técnica gerou três mapas temáticos, um para cada data.

Técnica 2 - Classificação supervisionada da imagem TM/Landsat5 de 15-8-2001, utilizando amostras coletadas diretamente na imagem, com o objetivo de criar condições de comparação com a técnica anterior (Técnica 1), proposta nesta pesquisa. A classificação realizada para o segundo processo utilizou o mesmo classificador da anterior (Técnica 1 - Mahalanobis), de maneira a se manter o mesmo padrão de classificação das imagens. A Técnica 2 foi adotada, buscando reproduzir os procedimentos operacionais de uma classificação usual, baseada somente nas variações espectrais. Neste caso, a imagem do dia 15-8-2001 foi a escolhida para comparações, baseando-se nos resultados encontrados por MOREIRA et al. (2004), em que os autores constataram que o melhor período para identificação da cultura de café ocorreu nas imagens obtidas durante os meses secos na região (maio até outubro). 
Para os processamentos da classificação utilizando a Distância de Mahalanobis, no software ENVI 4.0, foi necessário o ajuste da variável correspondente ao erro de máxima distância, para o valor 2. Os valores maiores que dois superestimavam a área, e os valores menores que dois subestimavam a área, tendo como parâmetro de referência a máscara.

Por fim, a Técnica 3 foi realizada por meio de uma composição temática, representada pela soma dos pixels (operação booleana "<or $>$ ") resultantes das classificações obtidas pela aplicação da Técnica 1, a qual gerou três imagens temáticas de áreas de café. O objetivo foi gerar um novo mapa, com as informações contidas nas imagens temáticas.

A qualidade das informações resultantes da aplicação das Técnicas 1;2 e 3 foi realizada aplicando a técnica da estatística Kappa, através da Exatidão Global, representada pela operação com os dados referentes à diagonal principal da Matriz de Erro, e o coeficiente Kappa (COHEN, 1960) que é muito utilizado como medida de exatidão de uma classificação temática e definido pelas eqs.(2) e (3), respectivamente:

$$
E G=\left(\frac{A}{N}\right) * 100
$$

em que,

EG - exatidão global;

A - acerto geral (pontos amostrais na diagonal principal), e

$\mathrm{N}$ - número de pontos amostrais.

$$
K=\frac{N \sum_{i=1}^{r} x_{i i}-\sum_{i=1}^{r}\left(x_{i+} * x_{+i}\right)}{N^{2}-\sum_{i=1}^{r}\left(x_{i+} * x_{+i}\right)}
$$

em que,

K - coeficiente Kappa de concordância;

$\mathrm{N}$ - número de observações, pontos amostrais;

$\mathrm{r}$ - número de linhas da matriz de confusão;

$\mathrm{X}_{\mathrm{ii}}$ - observações na linha i e coluna $\mathrm{i}$;

$\mathrm{X}_{\mathrm{i}+}$ - total da marginal da linha $\mathrm{i}$, e

$\mathrm{X}_{+\mathrm{i}}$ - total da marginal da coluna $\mathrm{i}$.

Para construir a matriz de erros, foi executada a seguinte operação sobre os mapas temáticos resultantes, denominados Técnica 1, Técnica 2 e Técnica 3: foram espacializados 100 pontos amostrais por meio da amostragem randômica aleatória, função disponível no software ENVI 4.0, tendo como espaço amostral o município de Guaxupé. O mesmo conjunto de pontos foi utilizado nos mapas dos três processos.

Desta maneira, a metodologia pretende contribuir com o aumento da acurácia na classificação de áreas com café, independentemente das condições de tipo de plantio, mas considerando, de forma mais efetiva, as condições de iluminação.

\section{RESULTADOS E DISCUSSÃO}

\section{Componentes Principais na escolha das amostras}

Na Figura 1, estão contidos os gráficos resultantes, após a aplicação da ACP, sobre as três imagens TM/Landsat 5 (quadros $a, b$ e $c$ ). Nestas figuras, estão contidas as amostras referentes a oito grupos, porém as amostras efetivamente selecionadas formaram três grupos de dados de cada tipo de cultura de café devido à sobreposição de pontos no interior da "nuvem".

Os grupos utilizados para a seleção de amostras foram em número menor que os grupos extraídos das imagens de FI devido à exclusão das classes de Café Adensado Pouco Iluminado (ADEN_PI) e Café Adulto Pouco Iluminado (ADUL_PI), onde as amostras se confundiram com as 
classes de Café Adensado Iluminado (ADEN_IL) e Café Adulto Iluminado (ADUL_IL), respectivamente. Na Tabela 3, mostram-se as classes finais consideradas em função da análise da quantidade de pixels disponíveis para serem utilizadas na classificação da ACP.
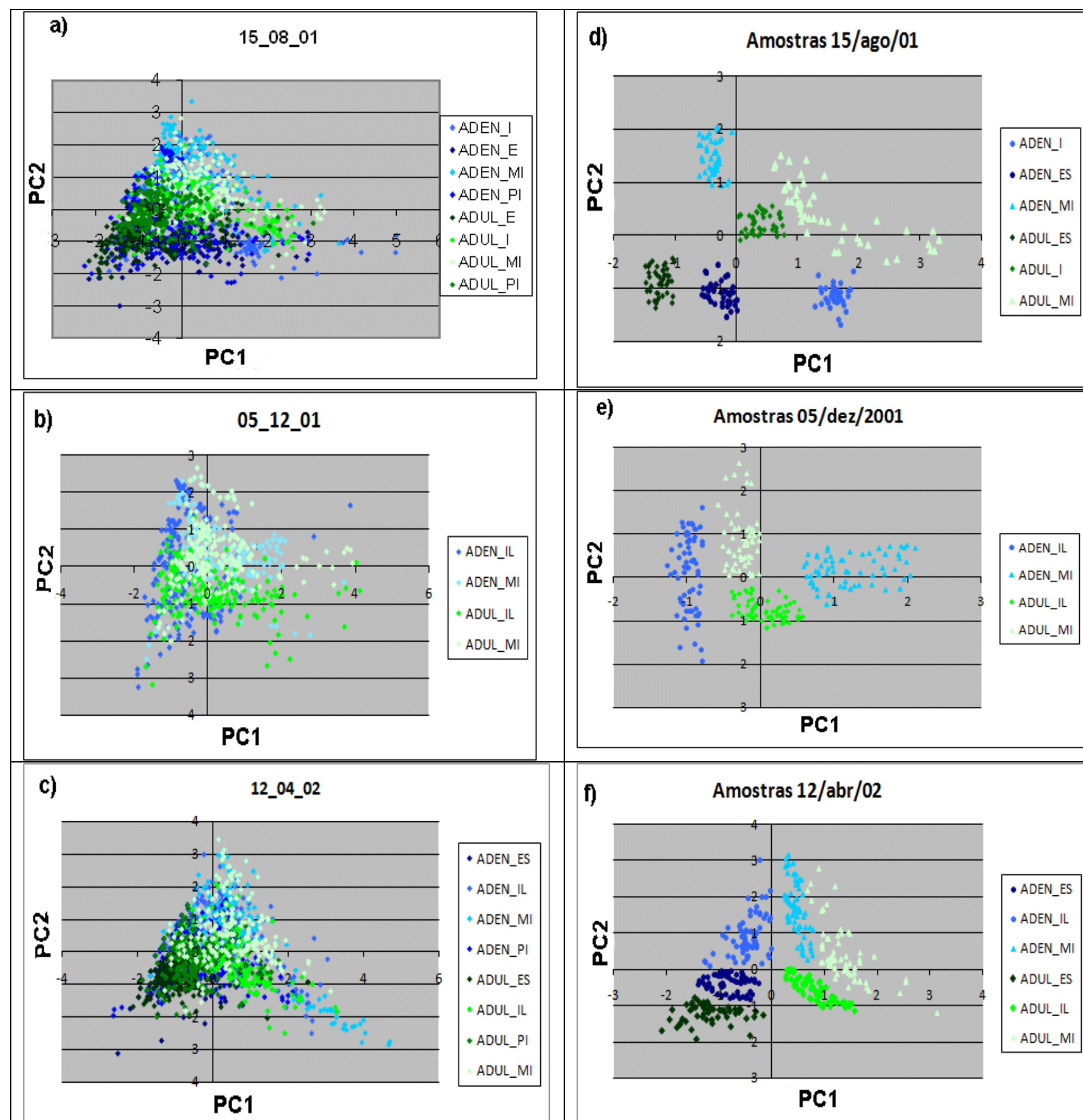

e)
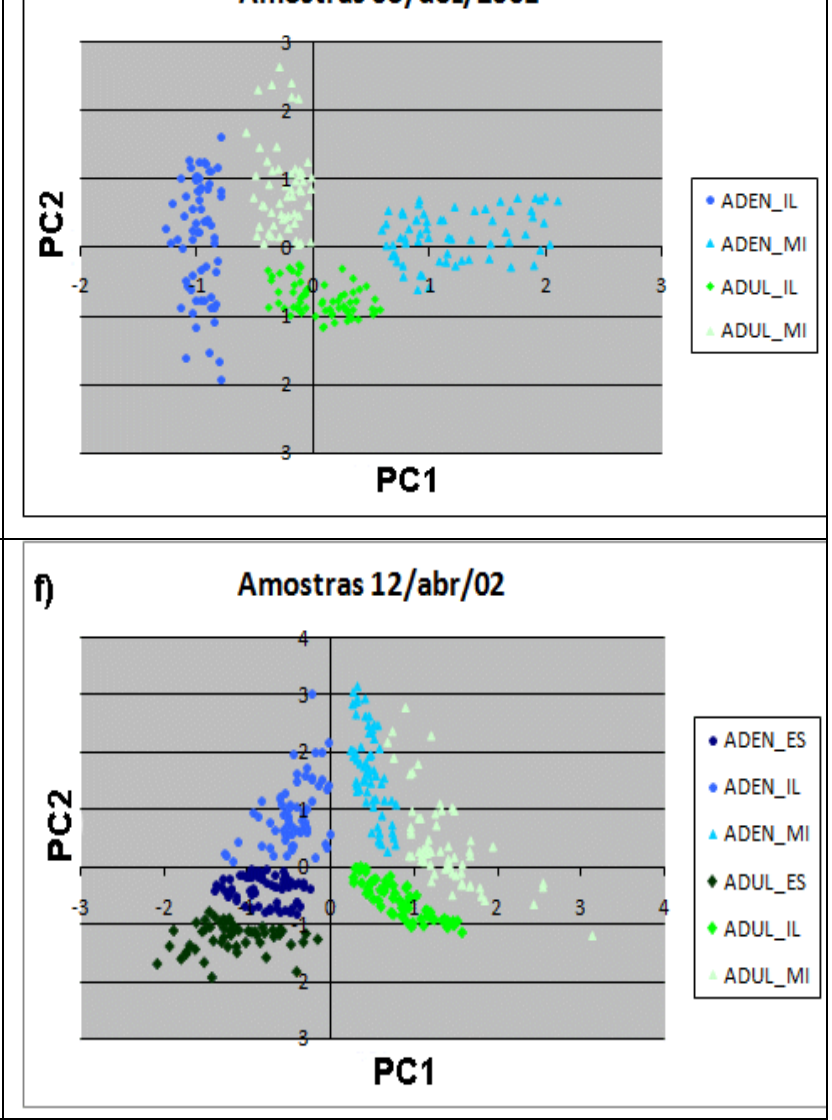

FIGURA 1. Gráficos resultantes da aplicação da ACP $(a, b$ e $c)$ e gráficos resultantes da seleção de amostras de treinamento $(d, e$ e $f)$, utilizadas na aplicação da Técnica 1 . Results from PCA application Method $(a, b, c)$ and training samples (d,e and f) used in Method 1.

As amostras extraídas dos grupos de pontos formados pelos fatores $f 1$ e $f 2$ perfizeram um total de 65 amostras por grupo. Esta quantidade foi definida em função da localização dessas amostras em relação ao conjunto, ou seja, amostras destacadas dentro do grupo a que pertencem, e destacadas, também, das amostras referentes a outros grupos, conforme CANAVESI (2008). Os gráficos resultantes permitiram a visualização da distribuição das "nuvens" de pontos que receberam cores diferentes para facilitar a identificação. Os gráficos referentes à Figura 1, nos 
quadros $d$ e $f$, apresentam as seis classes possíveis de iluminação, e o quadro $e$ apresenta o gráfico contendo as quatro classes possíveis selecionadas.

TABELA 3. Distribuição dos grupos amostrais selecionados após a aplicação da ACP. Distribution of sampling per group after PCA application Method.

\begin{tabular}{lcccc}
\hline \multicolumn{1}{c}{ Tipo de café } & \multicolumn{4}{c}{ Data do Fator de Iluminação } \\
\cline { 2 - 5 } \multicolumn{1}{c}{ Iluminação } & FI & FI & FI & \multirow{2}{*}{ Cores adotadas } \\
\hline Adensado escuro (ADEN_ESC) & $15-8-2001$ & $5-12-2001$ & $12-4-2002$ & \\
Adensado iluminado (ADEN_IL) & $\mathrm{X}$ & & $\mathrm{X}$ & \\
Adensado muito iluminado (ADEN_MI) & $\mathrm{X}$ & $\mathrm{X}$ & $\mathrm{X}$ & \\
Adulto escuro (ADUL_ES) & $\mathrm{X}$ & $\mathrm{X}$ & $\mathrm{X}$ & \\
Adulto iluminado (ADUL_IL) & $\mathrm{X}$ & $\mathrm{X}$ & $\mathrm{X}$ & \\
Adulto muito iluminado (ADUL_MI) & $\mathrm{X}$ & $\mathrm{X}$ & $\mathrm{X}$ & \\
\hline
\end{tabular}

\section{Análise por Componentes Principais (ACP)}

Os resultados referentes à aplicação da ACP foram definidos pelos valores das componentes, e pelos dados estatísticos referentes aos autovalores e autovetores, que servem como fatores de ponderação e definem a contribuição de cada banda original para uma componente principal (CP). Conforme a Tabela 4, que contém os resultados da análise realizada na imagem de 15-8-2001 foram escolhidas as duas primeiras componentes, PC1 e PC2, pois apresentaram o valor acumulado de $94,358 \%$ das informações iniciais.

TABELA 4. Valores estatísticos resultantes da ACP referentes às amostras de 15-8-2001 - Técnica 1. Sample statistics values from PCA in 08/15/01.

\begin{tabular}{cccc}
\hline & \multicolumn{3}{c}{ Variância Total (15-8-2001) } \\
\hline \multirow{2}{*}{ Componentes } & \multicolumn{3}{c}{ Autovalores iniciais } \\
\cline { 2 - 4 } & Total & \% de variação & \% acumulado \\
\hline 1 & 4,608 & 76,806 & 76,806 \\
2 & 1,053 & 17,552 & 94,358 \\
3 & 0,179 & 2,978 & 97,337 \\
4 & 0,100 & 1,670 & 99,007 \\
5 & 0,360 & 0,607 & 99,614 \\
6 & 0,023 & 0,386 & 100,000 \\
\hline
\end{tabular}

A aplicação da técnica de ACP apresentou algumas características relevantes quanto à distribuição das amostras nas "nuvens" resultantes. A forte correlação existente entre os dados contidos nas bandas multiespectrais é eliminada pela aplicação da técnica de ACP, em que, a redundância das informações é removida, porém constatou-se que as "nuvens" de pontos apresentaram forte tendência à concentração no entorno do centroide a partir da data de 15-8-2001 (Figura 1, quadros $a, b$ e $c$ ), aumentando a densidade em 5-12-2001 e 12-4-2002, provocando maior intersecção. Portanto, as amostras (65 para cada grupo) foram identificadas mais claramente e, assim, retiradas da imagem do dia 15-8-2001, pois era nela que havia menos confusão entre os grupos.

\section{Classificação supervisionada}

No mapa baseado na aplicação da Técnica la (15-8-2001), mostrado na Figura 2, as áreas de café ADEN_MI, ADUL_MI e ADUL_IL, após análise visual, foram classificadas de forma bastante precisa quanto à localização, em relação aos polígonos de café da máscara, caracterizando, assim, a possibilidade de utilização do método proposto, para a localização do cafeeiro iluminado e muito iluminado. Na maioria dos polígonos, detectou-se a ausência da classe ADEN_IL (café adensado 
iluminado), tendo como provável causa a quantidade de polígonos levantados em campo (97), comparados com o número de polígonos cadastrados da classe café adulto (1291), comprovando a baixa quantidade de área plantada com esta característica de espaçamento.

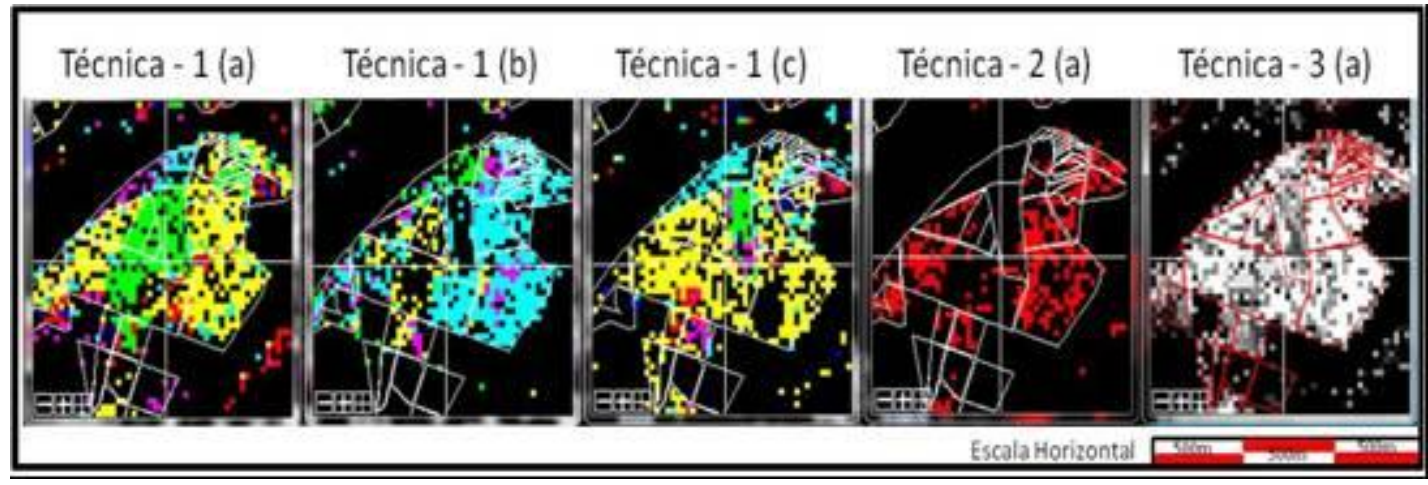

FIGURA 2. Quadro comparativo dos resultados da aplicação das técnicas de classificação e limites dos talhões. Comparative results from classification techniques and fields limits.

No mapa temático baseado na Técnica lb (5-12-2001), Figura 2, mostrou-se uma redução das classes de iluminação ocorrida na imagem devido ao grande ângulo de elevação solar correspondente, estabelecendo um novo cenário para o resultado da classificação supervisionada nesta data.

Nesta classificação, as classes ADUL_MI e ADEN_IL destacaram-se, porém, com uma quantidade de pixels menor na classificação, sendo que, em alguns setores, não houve identificação de áreas de café como aquelas classificadas na data 15-8-2001. O grande ângulo de elevação solar na imagem generalizou a iluminação nos locais que foram classificados de forma mais detalhada na imagem de 15-08-01 (Técnica la), conforme pode ser visto na Figura 2, comparando-se a Técnica la (15-8-2001) com a Técnica lb (5-12-2001).

Na aplicação da Técnica 1c (12-4-2002), em função da leve redução do ângulo de elevação solar, quando comparado com a imagem de 15-8-2001, as classes de iluminação utilizadas como amostras para a classificação (seis classes) na imagem de 12-4-2002 resultaram em uma imagem com características muito próximas entre as duas datas.

A escolha das amostras utilizando a metodologia usual adotada na aplicação da Técnica 2 (escolha das amostras de treinamento conforme a experiência de campo do observador baseado no comportamento espectral), que utilizou a imagem de 15-8-2001, conforme Figura 2, Técnica 2a, resultou na não classificação de pixels considerados escuros (ADEN_ES, ADUL_ES) e regiões de cafeeiro que foram identificadas nas aplicações da Técnica 1.

Resultante da operação de composição, o mapa temático baseado na Técnica 3 apresentou o melhor resultado no que diz respeito à localização no mapeamento das áreas cafeeiras, como pode ser observado na Técnica 3a da Figura 2.

A composição formada pelos mapas resultantes da aplicação da Técnica 1 agrupou os pixels identificados como café, em condições de diferentes ângulos de elevação solar, aumentando o preenchimento dos pixels no interior dos limites dos talhões de café utilizados como referência de localização da cultura cafeeira plantada no município de Guaxupé.

A mesma composição trouxe, também, a concentração dos pixels localizados em áreas classificadas como não café, mas que terminaram por trazer informações bastante importantes sob o ponto de vista de iluminação, ou seja, algumas características de iluminação utilizadas como identificadores de áreas de café localizaram áreas de mata densa e mata ciliar, tendo o mapa resultante da aplicação da Técnica 3, trazido à mostra, o destaque da concentração dessas informações, conforme explicado a seguir. 
Durante a escolha dos intervalos de fatiamento nas imagens do FI, foi possível a definição de todas as classes de iluminação, levando-se em consideração os talhões de café cadastrados em campo. Porém, durante a escolha das amostras geradas pela aplicação da ACP, as amostras referentes às classes pouco iluminada (ADUL_PI e ADEN_PI) e escura (ADUL_ES e ADEN_ES), confundiram-se no interior da "nuvem" de pontos, tornando difícil o isolamento das amostras ADUL_PI e ADEN_PI, atribuindo, então, prioridade para a coleta das amostras da classe escura (ADUL_ES e ADEN_ES).

O objetivo de se optar pela seleção de amostras da classe escura (ADUL_ES e ADEN_ES), limite inferior do fatiamento, foi o de buscar informações relevantes na zona escura, que tem sido descrita na literatura especializada como a área de maior concentração de confusão espectral, principalmente em relevo montanhoso.

Os mapas temáticos resultantes da aplicação da classificação supervisionada (Técnica 1 nas imagens de 15-8-2001 e 12-4-2002) apresentaram para as áreas de café, na classe escura (ADUL_ES e ADEN_ES), áreas correspondentes à mata densa e mata ciliar (próxima dos cursos d'água), confirmando a confusão espectral, nestes casos, conforme apresentado na Figura 3 (linha na cor amarela).

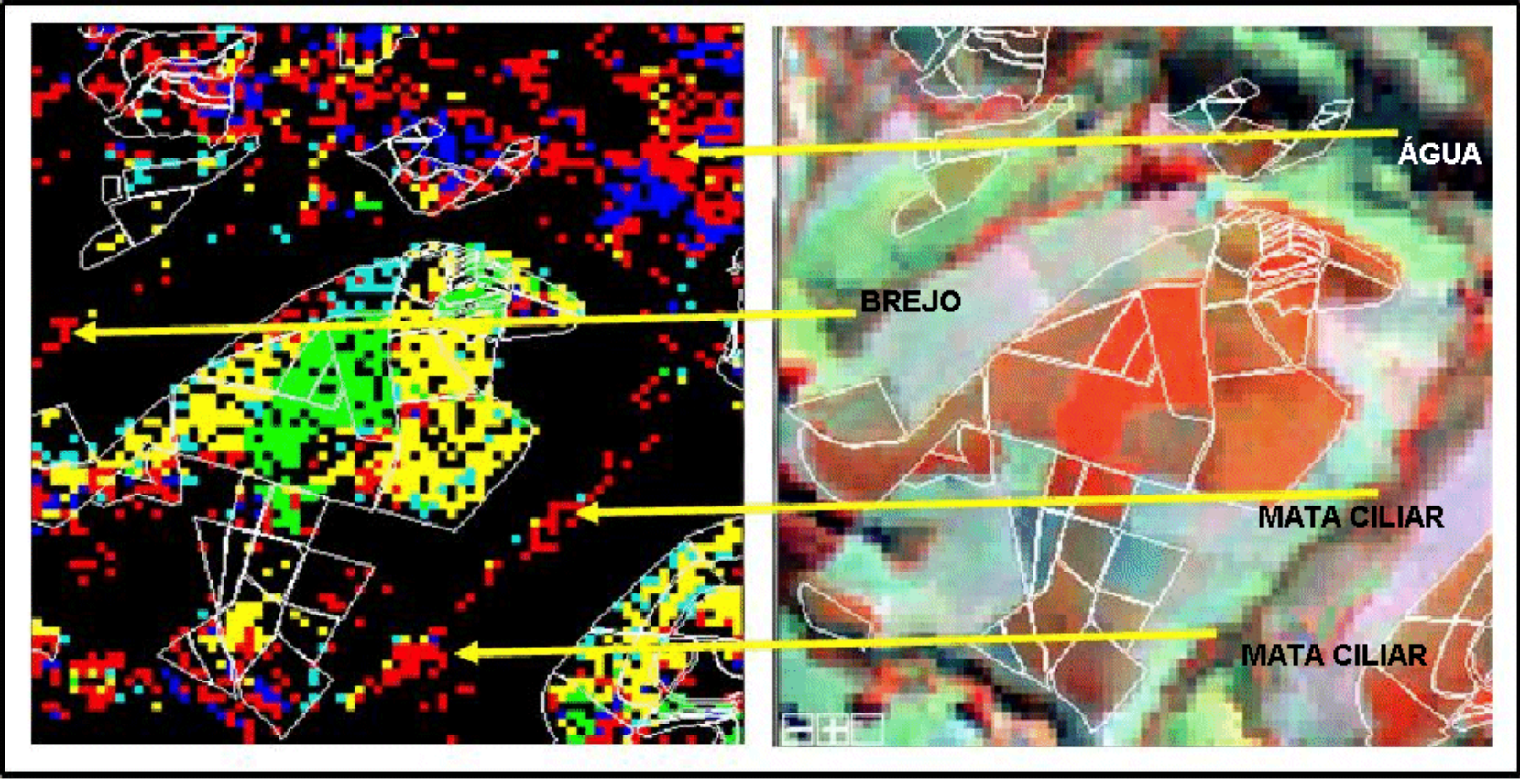

FIGURA 3. Recortes da imagem TM/Landsat 5 de 15-8-2001 (direita) e os correspondentes recortes do mapa temático gerado pela classificação, utilizando-se da Técnica 1 (esquerda). Zoom in the Landsat images in $08 / 15 / 01$ ( right) and zoom from thematic map after Method 1 classification (left).

\section{Validação dos dados}

Os resultados finais obtidos pela aplicação da técnica de classificação supervisionada passaram pela análise da validação dos dados, conforme tem sido adotado como procedimento na literatura especializada, de forma a se estabelecerem parâmetros que orientem quanto à qualidade do processo aplicado. Os resultados estatísticos obtidos na validação dos dados nesta pesquisa são baseados nos valores obtidos pela Matriz de Erro, pela Exatidão Global e pelo coeficiente Kappa.

Na matriz de Erro construída com os valores amostrais referentes à aplicação da Técnica 1 sobre a imagem de 15-8-2001, apresentada na Tabela 5, pode-se constatar que o número de amostras referentes à classe Café (13) foi bastante inferior ao número de amostras classificadas 
como Não Café (65), o que confirma a grande variação entre as áreas ocupadas por lavouras de café em relação à área do município.

TABELA 5. Comparativo dos resultados da validação das classificações. Comparative results from the classification validation.

\begin{tabular}{|c|c|c|c|c|c|c|c|}
\hline \multirow{2}{*}{$\begin{array}{l}\text { Técnica } \\
\text { Aplicada }\end{array}$} & \multirow{2}{*}{ Máscara } & \multirow{2}{*}{ Café } & \multirow{2}{*}{ Não Café } & \multicolumn{2}{|c|}{ Erros } & \multirow{2}{*}{$\begin{array}{c}\text { Exatidão } \\
\text { Global }\end{array}$} & \multirow{2}{*}{$\begin{array}{l}\text { Coef. } \\
\text { Kappa }\end{array}$} \\
\hline & & & & Inclusão & Omissão & & \\
\hline \multirow{3}{*}{$\begin{array}{l}\text { Técnica } 1 \\
15-8-2001\end{array}$} & Café & 13 & 15 & 0,536 & 0,35 & & \\
\hline & Não café & 7 & 65 & 0,097 & 0,188 & 0,78 & 0,40 \\
\hline & Soma & 20 & 80 & & & & \\
\hline \multirow{3}{*}{$\begin{array}{l}\text { Técnica } 1 \\
5-12-2001\end{array}$} & Café & 6 & 4 & 0,400 & 0,714 & & \\
\hline & Não café & 15 & 75 & 0,167 & 0,051 & 0,81 & 0,29 \\
\hline & Soma & 21 & 79 & & & & \\
\hline \multirow{3}{*}{$\begin{array}{l}\text { Técnica } 1 \\
12-4-2002\end{array}$} & Café & 6 & 22 & 0,786 & 0,250 & & \\
\hline & Não café & 2 & 70 & 0,028 & 0,239 & 0,76 & 0,24 \\
\hline & Soma & 8 & 92 & & & & \\
\hline \multirow{3}{*}{$\begin{array}{l}\text { Técnica } 2 \\
15-8-2001\end{array}$} & Café & 5 & 3 & 0,375 & 0,706 & & \\
\hline & Não café & 12 & 80 & 0,130 & 0,036 & 0,85 & 0,33 \\
\hline & Soma & 17 & 83 & & & & \\
\hline \multirow{3}{*}{ Técnica 3} & Café & 20 & 18 & 0,474 & 0,048 & & \\
\hline & Não café & 1 & 61 & 0,016 & 0,228 & 0,81 & 0,56 \\
\hline & Soma & 21 & 79 & & & & \\
\hline
\end{tabular}

O número de amostras correspondente aos acertos na classe Café representou $65 \%$ do total de amostras referentes à classe, representando um número 1,87 superior ao número de amostras não classificadas.

O valor referente ao resultado da Exatidão Global reflete o alto percentual de acertos na classificação, porém foi fortemente influenciado pelos acertos das amostras da classe Não Café. Quanto ao coeficiente Kappa, que reflete a qualidade da classificação de uma maneira mais abrangente sob o aspecto de utilizar os valores de inclusão e exclusão das classes, espelhou a diferença entre as áreas de lavoura de café (6002 ha) e a área total do município utilizada pela classe Não Café (28.600 ha), apresentando um baixo valor.

A Técnica 1 (5-12-2001) mostra, nos resultados apresentados na Tabela 4, forte redução das amostras classificadas na classe Café, localizando somente 6 amostras $(28,6 \%)$ do total. A matriz apresenta, também, o aumento do número de amostras de café localizadas dentro dos polígonos (15), mas não classificada pelo processamento $(71,4 \%)$. A utilização de um número menor de classes de iluminação para a imagem FI, de 5-12-2001, foi comprovada pela forte redução das amostras referentes à mata (erro de inclusão na classe Café) representada por 5,06\% das amostras da classe Não Café (quatro amostras). Isto corrobora os resultados anteriores deste trabalho, de que valores de classes de Fator de Iluminação baixos contribuem para a identificação de mata e mata ciliar.

O pior desempenho representado pela validação dos dados refere-se à classificação adotando-se a Técnica 1 para a imagem de 12-4-2002, em que os percentuais referentes às amostras da classe Café classificados corretamente representaram 75\% das amostras (seis amostras) desta classe, porém representando somente $6 \%$ do total de amostras. Em contrapartida, a quantidade de amostras que possibilitou a identificação de mata (22 amostras) representou $23,91 \%$ das amostras da classe Não Café. E o percentual de acerto na classificação das amostras da classe Não Café ficou na casa de 76,09\% (70 amostras). 
A Técnica 2 mostrou um fraco desempenho na classificação, mesmo utilizando a imagem de 15-8-2001, que ofereceu os melhores resultados na classificação, quando comparados com os valores obtidos na classificação das outras duas datas (5-12-2001 e 12-4-2002). O bom desempenho, 0,85 de Exatidão Global, deveu-se à boa perfomance na discriminação da Classe Não Café. De fato, o erro de omissão de 0,706 para a classe Café, neste tipo de abordagem, foi muito alto, diminuindo sua efetividade. Por outro lado, se tivesse sido realizado o procedimento multitemporal para a Técnica 2, talvez os resultados pudessem ser melhores. Isto não foi testado neste trabalho.

Os valores percentuais obtidos mostram, neste caso específico, que a Técnica 3 obteve os melhores resultados, em que o número de amostras classificadas como Café atingiu o percentual de 95,24\% (20 amostras) das amostras da classe, deixando de classificar somente 4,76\% das amostras (uma amostra). Este resultado comprova o bom desempenho, também, da distribuição das amostras da classe Não Café. Neste caso, $77,21 \%$ das amostras foram classificadas corretamente (61 amostras), e $22,79 \%$ das amostras identificaram áreas constituídas de mata densa ou mata ciliar (18 amostras). A efetividade da metodologia é corroborada pelo trabalho de TISOT et al.(2007) que, analisando dados do ETM+ encontrou valores de 76,35\% de acerto para café, utilizando MaxVer, com erros de Comissão e Omissão de 11,33 e 23,65\%, respectivamente.

\section{Determinação de área nos mapeamentos}

Buscando a confirmação dos resultados apresentados nos processos de classificação e validação dos dados que, com base nos resultados de Exatidão Global e coeficiente Kappa, apresentaram valores que não retrataram com a eficiência usual a real situação da distribuição do cafeeiro na região devido à ocupação da área cafeeira ser muito menor em a área total do município.

Utilizando-se dos mapas resultantes da aplicação da classificação supervisionada, foram geradas imagens onde as áreas ocupadas pelo cafeeiro foram transformadas em valor igual a um (1), e as áreas de não café, iguais a zero (0). As cinco imagens resultantes (três mapas referentes à aplicação da Técnica 1, um mapa da Técnica 2 e o mapa relativo à aplicação da Técnica 3) foram multiplicadas, uma a uma, com a imagem gerada do mapa de vetores de café utilizado como referencial da área de plantio do município de Guaxupé.

O método adotado apresentou o percentual real do cafeeiro identificado no processo de classificação. Os valores das áreas de cada mapa podem ser visualizados na Tabela 6. Desta maneira, constatou-se que realmente a Técnica 3 foi a mais eficaz, possibilitando a identificação de $73,06 \%$ (4.385,70 ha) da área de café em relação à área total da máscara de café (6.002,64 ha), valor considerado ótimo para classificações de alvos em regiões montanhosas.

TABELA 6. Comparativo das áreas cafeeiras classificadas. Comparative chart of the coffee areas from the different methods used.

\begin{tabular}{ccc}
\hline Imagens & Área do Cafeeiro (ha) & Percentual de Classificação \\
\hline Técnica 1 - 15/08/01 & $2.975,67$ & $49,57 \%$ \\
Técnica 1 - 05/12/01 & $2.159,46$ & $35,97 \%$ \\
Técnica 1 - 12/04/02 & $2.297,79$ & $38,27 \%$ \\
Técnica 2 - 15/08/01 & $1.164,51$ & $19,40 \%$ \\
Técnica 3 - Composição & $4.385,70$ & $73,06 \%$ \\
\hline Referência & $6.002,64$ & $100,00 \%$ \\
\hline
\end{tabular}

\section{CONCLUSÕES}

A metodologia proposta nesta pesquisa, a utilização da Análise por Componentes Principais em conjunto com o Fator de Iluminação, confirma a possibilidade de maior eficácia na identificação do cafeeiro plantado em região montanhosa, quando comparada com métodos de classificação que não consideram o fator iluminação. 
A imagem utilizada na Técnica 3, que leva em consideração a soma (operação booleana) dos pixels encontrados por classificações supervisionadas, apresentou os resultados mais significativos, tanto no coeficiente Kappa quanto nos erros de omissão na classe Café e de inclusão na classe Não Café.

A amostragem utilizada não representou a área efetivamente classificada.

A Técnica 3, que leva em consideração a soma (operação booleana) dos pixels encontrados por classificações supervisionadas, retratou, mais efetivamente, os locais classificados como café, confirmando, acertadamente, a adoção do caráter de multitemporalidade na identificação da cultura do café.

\section{AGRADECIMENTOS}

À Cooxupé, especificamente ao Dr. Carlos Paulino da Costa (Presidente), Dr. Antônio Carlos Martins (Diretor Administrativo) e ao Eng ${ }^{\circ}$ Agrônomo Éder Ribeiro dos Santos, pelo apoio nas fases de coleta de dados e discussões dos resultados.

Ao Instituto Virtual FAPESP-Microsoft (projeto eFarms), pelo financiamento de parte do projeto.

\section{REFERÊNCIAS}

CAMARGO, A.P.; CAMARGO, M.B.P. Definição e esquematização das fases fenológicas do cafeeiro arábica nas condições tropicais do Brasil. Bragantia, Campinas, v.60, n.1. p.65-68, 2001.

CANAVESI, V. Aplicação de dados hiperespectrais na extração de informações qualitativas $e$ quantitativas de plantios florestais do gênero Eucalyptus spp. 2008. 145 f. Tese (Doutorado em Sensoriamento Remoto) - Instituto de Pesquisas Espaciais, São José dos Campos, 2008.

CANTY, M.J.; NIELSEN, A.A.; SCHMIDT, M. Automatic radiometric normalization of multitemporal satellite imagery. Remote Sensing of Environment, New York, v.91, n.3/4. p.441451, 2004.

COHEN, J.A. Coefficient of agreement for nominal scales. Educational and Psycological Measurement, Durham, v.20, n.1, p.37-46, 1960.

CORDERO-SANCHO, S.; SADER, S.A. Spectral analysis and classification accuracy of coffee crops using Landsat and a topographic-environmental model. International Journal of Remote Sensing, London, v.28, n.7. p.1.577-1.593, 2007.

LANG, R.H.; WELCH, R. Algorithm theoretical basis document for Aster digital elevation models, 1999. Disponível em: <http://eospso.gsfc.nasa.gov/eos_homepage/for_scientists/atbd/ docs/ASTER/atbd-ast-14.pdf>. Acesso em: 19 nov. 2006.

MERCANTE, E. Dinâmica espectral da cultura da soja ao longo do ciclo vegetativo e sua relação com a produtividade na região oeste do Paraná. 2008. $241 \mathrm{f}$. Tese (Doutorado em Planejamento e Desenvolvimento Rural Sustentável) - Faculdade de Engenharia Agrícola, Universidade Estadual de Campinas, 2008.

MOREIRA, M.A.; ADAMI, M.; RUDORFF, B.F.T. Análise espectral e temporal da cultura do café em imagens Landsat. Pesquisa Agropecuária Brasileira, Brasília, v.39, n.3, p.223-231, 2004.

NASCIMENTO, C.R.; ZULLO JR., J. Proposta metodológica de utilização de dados do sensor MODIS para correção atmosférica de imagens NOAA/AVHRR, visando à geração de imagens NDVI em modelos agrometeorológicos. In: CONGRESSO BRASILEIRO DE AGROMETEOROLOGIA, 14., 2005. Anais...

PONZONI, F.J.; SHIMABUKURO, Y.E. Sensoriamento remoto no estudo da vegetação. São José dos Campos: A. Silva Vieira Ed., 2007. 
RICHARDS, J.A.; JIA, X. Remote sensing digital image analysis: an introduction. 3.ed. New York: Springer: Verlag Berlin Heidelberg, 2006.

TISOT, D.A.; FORMAGGIO, A.R.; RENNÓ, C.D.; GALVÃO, L.S. Eficácia de dados Hyperion/EO-1 para identificação de alvos agrícolas: comparação com dados Etm+/Landsat-7. Engenharia Agrícola, Jaboticabal, v.27, n.2, p.511-519, 2007.

VIEIRA, T.G.C.; ALVES, H.M.R.; LACERDA, M.P.C.; VEIGA, R.D.; EPIPHANIO, J.C.N. Crop parameters for the evaluation of the spectral response of coffee (Coffea arabica) areas within the State of Minas Gerais, Brazil. São José dos Campos: INPE/DSR, 2004.

ZULLO JR., J. Correção atmosférica de imagens de satélite e aplicações. 1994. 190 f. Tese (Doutorado em Engenharia Elétrica) - Faculdade de Engenharia Elétrica, Universidade Estadual de Campinas, Campinas, 1994. 